\title{
Pólen coletado por Scaptotrigona depilis (Moure) (Hymenoptera, Meliponini), na região de Dourados, Mato Grosso do Sul, Brasil
}

\author{
Marcos G. Ferreiraํㅜㄹ Fátima C. D. Manente-Balestieri² \& José B. P. Balestieri²
}

${ }^{1}$ Programa de Pós-graduação em Entomologia e Conservação da Biodiversidade, Universidade Federal da Grande Dourados. Rodovia Dourados-Itahum, km

12, Caixa Postal 533, 79804-970 Dourados-MS, Brasil. mgfbio@yahoo.com.br

2Faculdade de Ciências Biológicas e Ambientais, Universidade Federal da Grande Dourados. crisdelazari@yahoo.com.br; pbalestieri@yahoo.com.br

\begin{abstract}
Pollen harvest by Scaptotrigona depilis (Moure) (Hymenoptera, Meliponini) in Dourados, Mato Grosso do Sul, Brazil. Pollen harvest by workers of Scaptotrigona depilis (Moure, 1942) were identified in the region of Dourados, Mato Grosso do Sul, during the period of September, 2006 to August, 2007. From the 42 pollen types collected, the more representative ones during this period were those from the Myrtaceae (Eucalyptus spp.) and Mimosaceae family. The peaks for the values of the trophic niche breadth $\left(\mathrm{H}^{`}\right)$ in September (2.32), November (2.29) and October (2.02) showed that these bees can be generalist, depending upon availabilities and characteristics of flower sources. The values for equitability $\left(\mathrm{J}^{\circ}\right)$ also showed more uniform use of pollen sources during these months. The meteorological features (temperature, relative humidity and rainfall) did not show a direct relation to equitability and niche breadth. Equitability and niche breadth seem to be more related to the daily or seasonal cycles of pollen and nectar production.
\end{abstract}

KEYWORDS. Diversity; floral resource; pollination; stingless bees.

RESUMO. Pólen coletado por Scaptotrigona depilis (Moure) (Hymenoptera, Meliponini), na região de Dourados, Mato Grosso do Sul, Brasil. Foram identificados os pólens coletados por operárias de Scaptotrigona depilis (Moure, 1942), na Região de DouradosMs, durante o período de setembro de 2006 a agosto de 2007. Ao todo foram coletados 42 tipos polínicos, sendo mais representativa em número de espécies, as famílias Myrtaceae (Eucalyptus spp.) e Mimosaceae. Os picos de valores de amplitude de nicho trófico (H') em Setembro $(2,32)$, Novembro $(2,29)$ e Outubro $(2,02)$ mostraram que essa abelha pode ser generalista, dependendo da disponibilidade e características das fontes florais. Os valores de equitatividade (J'), também indicam maior uniformidade de uso das fontes florais durante esses meses de máxima amplitude de nicho. Os fatores ambientais (temperatura, umidade relativa e pluviosidade) não mostraram relação direta com a equitatividade e amplitude do nicho trófico. Estas variáveis parecem estar mais relacionadas com os ciclos diários ou sazonais de produção de pólen e néctar.

PALAVRAS-CHAVE. Abelhas sem ferrão; diversidade; polinização; recurso floral.

A crescente devastação de habitats naturais tem aumentado a preocupação dos especialistas com os possíveis efeitos sobre as populações de abelhas nativas e, consequentemente, com o desenvolvimento de estratégias para a sua preservação (Matheson et al. 1996). Segundo Kerr et al. (1999) o Brasil possui a maior diversidade de abelhas sem ferrão do mundo, podendo representar 90\% dos polinizadores de um ecossistema, sendo assim, a preservação dos meliponíneos é também uma garantia de manutenção da base da cadeia alimentar. Além da manutenção da diversidade florística dos ecossistemas, um efeito direto da polinização por abelhas pode ser visto no aumento da produtividade de plantas cultivadas, através da introdução de ninhos em áreas agrícolas (Roubik 1995).

As relações dos meliponineos com as flores podem ser analisadas de maneira indireta e prática através da análise polínica do alimento transportado pelas campeiras para as colônias. E essa abordagem permite estimar o espectro de fontes florais e sua atratividade relativa sobre as colônias, em dado período ou hábitat (Imperatriz-Fonseca et al. 1993).

De acordo com Ramalho et al. (2007), o hábito generalista em meliponíneos é considerado uma necessidade básica e, portanto, aceito como padrão entre essas abelhas eusociais da família Apidae, com grandes colônias perenes, altas taxas de produção de prole e que precisam de muito alimento ao longo de todo ano.
No Mato Grosso do Sul são poucos os estudos do hábito alimentar das abelhas, com uso de grãos de pólen. Sendo a região de Dourados caracterizada por áreas cultivadas extensivas, sofrendo inúmeras perturbações ambientais que causam grande decréscimo nos recursos florais nativos, tornase importante conhecer os nichos tróficos dos polinizadores locais, com especial atenção às abelhas nativas para entender como elas estão respondendo a essas mudanças.

Este trabalho teve como objetivo, identificar as fontes florais de pólen utilizadas por Scaptorigona depilis (Moure, 1942), no intuito de conhecer como muda a amplitude do nicho trófico ao longo do ano e qual a importância relativa das fontes florais para essa abelha nativa.

\section{MATERIAL E MÉTODOS}

\section{Descrição do local}

O estudo foi realizado no Laboratório de Abelhas Nativas (LAN) na unidade II da Faculdade de Ciências Biológicas e Ambientais da UFGD, localizado nas coordenadas: $22^{\circ}$ 12' $16^{\prime}$ 'de latitude Sul e $54^{\circ} 48$ ' 20" de longitude Oeste em Dourados/MS. Numa área de 90 hectares nos arredores do LAN, Composta por uma vegetação secundária, formada principalmente por plantas ornamentais nativas e exóticas (Araliaceae, Leguminosas, Bignoniaceae, Bombacaceae, 
Myrtaceae, etc), culturas agrícolas de áreas experimentais (soja, algodão e milho) plantas medicinais e pomar, sendo seu entorno caracterizado por pastagens, culturas e vegetação típica de cerrado. A topografia do local é plana e o solo, originalmente sob a vegetação do Cerrado, é classificado como latossolo vermelho distroférrico de textura argilosa (Embrapa 1999). A altitude é de $464 \mathrm{~m}$ e, segundo Zavatini (1992), o regime pluviométrico é do tipo tropical, com índices médios superiores a $1600 \mathrm{~mm} / \mathrm{ano}$, com duas estações: a chuvosa que vai de outubro a março e a seca de abril a setembro. Dezembro, janeiro e fevereiro são os meses mais chuvosos e junho, julho e agosto, os mais secos.

Obtenção, preparação e identificação das amostras.

Foi utilizada uma colônia de Scaptotrigona depilis localizada no interior do Laboratório de Abelhas Nativas (LAN). Essa abelha é considerada uma espécie agressiva e bastante comum, cujos habitats e nicho ecológico compreendem desde o Rio Grande do Sul até o México. S. depilis vive em ocos de árvores e as colônias são bastante populosas, chegando à cerca de 2.000 a 50.000 abelhas (Nogueira-Neto 1970).

Para a obtenção das amostras foi utilizada uma coleta diária de no mínimo três abelhas campeiras na entrada do ninho em horários alternados, obedecendo à constância na coleta de pólen. As cargas polínicas foram retiradas das corbículas com o auxilio de um estilete e em seguida as amostras de pólen foram colocadas em frascos contendo ácido acético glacial. Após 24 hs, foram submetidas ao processo de acetólise, segundo o método descrito por Erdtman (1960), para preparação dos grãos de pólen. Os mesmos foram avaliados morfometricamente com uma ocular micrométrica, com aumento de 1000x (imersão) e mensurados de acordo com (Erdtman 1952). A identificação dos grãos de pólen foi realizada por comparação com lâminas da palinotecareferência do LAN/FCBA, com base nas características morfológicas: tamanho, unidade polínica, tipo de abertura, forma e escultura da parede. As identificações também foram feitas por consulta à literatura especializada (Absy 1975; Roubik \& Moreno 1991; Salgado-Labouriau 1973). Os parâmetros ambientais (temperatura e pluviosidade) foram obtidos na da Estação Meteorológica Automática da Embrapa Agropecuária Oeste em Dourados-MS.

\section{Análises estatísticas}

Para os cálculos estatísticos foram consideradas amostras mensais que levou em conta o número de ocorrência dos tipos polínicos durante o mês, sendo possível assim, obter os índices mensais de amplitude do nicho trófico e equitatividade.

A amplitude do nicho trófico foi calculada pelo índice de diversidade (H') de Shannon \& Weaver (1949) que leva em consideração a proporção dos tipos polínicos encontrados nas amostras mensais, indicando uma especificidade maior de coleta à medida que $\mathrm{H}^{\prime}$ diminui: $\mathrm{H}^{\prime}=\sum$ (pi.In pi), onde $\mathrm{H}^{\prime}$ é $\mathrm{o}$ índice de diversidade, pi é a proporção de cada tipo polínico encontrado nas amostras mensais e In o logaritmo natural.

A equitatividade, que indica o grau de uniformidade de coleta de pólen nas espécies vegetais visitadas pelas abelhas no mês considerado, foi calculada pelo índice J' Pielou (1977). Neste caso J' =H'/H' max, sendo H' o índice de diversidade e H'max o logaritmo neperiano do número total de tipos polínicos presentes na amostra. $O$ índice $J$ ' pode variar de 0 a 1, ou seja, de uma utilização heterogênea a uma utilização homogênea dos recursos.

\section{RESULTADOS E DISCUSSÃO}

Foram visitadas 42 espécies de plantas por Scaptotrigona depilis no período de setembro de 2006 a agosto de 2007. As famílias Myrtaceae e Mimosaceae foram as mais exploradas em número de espécies (Tabela I). Espécies de Mimosa (Mimosaceae) e Eucalyptus (Myrtaceae) estão presentes na maioria dos levantamentos de flora visitada por meliponineos no sudeste do Brasil (Imperatriz-Fonseca et al. 1989; Ramalho et al. 1989, 1990). A alta freqüência do pólen de Myrtaceae na dieta de $S$. depilis corrobora resultados de estudo de hábito alimentar com várias espécies de meliponineos em diferentes regiões do Brasil (Absy et al. 1984; Kleinert-Giovanini \& Imperatriz-Fonseca 1987; Ramalho 1990; Ramalho et al.1991, 2007; Wilms \& Wiechers 1997). Marques-Souza et al. (2007) trabalhando com amostras de pólen de Scaptorigona fulvicutis Moure, 1964, na Amazônia Central verificaram a coleta em 97 espécies de plantas distribuídas em 73 gêneros e 36 famílias, dentre as quais, Leguminosae (Mimosoidea) e Myrtaceae foram às famílias mais representativas.

Dentre as Myrtaceae, Eucalyptus sp. 1 foi comum ao longo de todo o período alcançando 31,6 \% do total dos grãos de pólen coletados, o segundo tipo polínico mais freqüente nas amostras foi Schefflera morototoni (Aubl.) Decne. \& Planch, que chegou a representar 13,2\% (Tabela I). Apesar da maior preferência por esses dois tipos polínicos, outras fontes florais tiveram presença bastante acentuada em meses isolados, atingindo porcentagens de coletas expressivas, como: Eucalyptus sp.3 com 8,2\%, Eucalyptus sp. 2 com 6,7\%, Myrtaceae tipo 3 com 5,2\% e Solanum sp. com 4,1\%. Outros tipos polínicos totalizaram 31\% (Tabela I). A preferência de S. depilis e outras espécies de Scaptotrigona pelo pólen de Eucalyptus sp. também foi verificada por Ramalho (1990), que relacionou essa preferência à alta concentração de pólen nas copas destas árvores e aos mecanismos específicos de forrageio que levam a alta concentração de campeiras em poucas fontes de pólen mais produtivas.

Com relação às características polínicas, foi possível notar que os grãos de pólen mais representativos nas amostras de $S$. depilis tinham tamanho médio. No que diz respeito à ornamentação da parede polínica, a escolha foi generalizada, porém com maior número de grãos de pólen com superfície psilada. Marques-Souza et al. (2002) trabalhando com espécies de abelhas nativas da Amazônia Central, não encontraram evidência de que as características morfológicas do pólen (tamanho, forma e ornamentação) estivessem influenciando as coletas das abelhas estudadas.

As características das flores (cor, tamanho, odor, forma e tipos de antera) e hábitos de coleta, juntamente com 
Tabela I. Distribuição mensal e percentual dos tipos polínicos coletados por Scaptotrigona depilis durante o período de setembro de 2006 a agosto de 2007 no Campus da UFGD, Dourados, MS.

\begin{tabular}{|c|c|c|c|c|c|c|c|c|c|c|c|c|c|}
\hline \multirow[b]{2}{*}{ Familia/Espécie } & \multicolumn{3}{|c|}{2006} & \multicolumn{9}{|c|}{2007} & \multirow[b]{2}{*}{$\%$} \\
\hline & set & out & nov & dez & jan & fev & mar & $\mathrm{abr}$ & mai & jun & jul & Ago & \\
\hline \multicolumn{14}{|l|}{ Anacardiaceae } \\
\hline Astronium sp. & $\mathrm{x}$ & & & & & & & & & & & & 0,3 \\
\hline \multicolumn{14}{|l|}{ Araliaceae } \\
\hline Schefflera morototoni (Aubl.) Decne. \& Planch & $\mathrm{x}$ & $\mathrm{x}$ & $\mathrm{x}$ & $\mathrm{x}$ & & $\mathrm{x}$ & $\mathrm{x}$ & $\mathrm{x}$ & $\mathrm{x}$ & $\mathrm{x}$ & $\mathrm{x}$ & & 13,2 \\
\hline Araliaceae tipo & & & & & & & & $\mathrm{x}$ & & & & & 0,3 \\
\hline \multicolumn{14}{|l|}{ Asteraceae } \\
\hline Baccharis sp. & & & $\mathrm{x}$ & $\mathrm{x}$ & $\mathrm{x}$ & & & $\mathrm{x}$ & & $\mathrm{x}$ & & & 1,5 \\
\hline \multicolumn{14}{|l|}{ Bignoniaceae } \\
\hline Tabebuia sp. & $\mathrm{x}$ & & & & & & & & $\mathrm{x}$ & $\mathrm{x}$ & & $\mathrm{x}$ & 0,9 \\
\hline \multicolumn{14}{|l|}{ Bombacaceae } \\
\hline Ceiba boliviana Britten \& Baker f. & & & & & & & & $\mathrm{x}$ & $\mathrm{x}$ & & & & 0,4 \\
\hline \multicolumn{14}{|l|}{ Euphorbiaceae } \\
\hline Sapium glandulatum (Vell.) Pax & $\mathrm{x}$ & $\mathrm{x}$ & $\mathrm{x}$ & & & & & & & & & & 3,2 \\
\hline Caesapiniaceae & & & & & & & & & & & & & \\
\hline Caesalpinia peltophoroides Benth. & $\mathrm{x}$ & $\mathrm{x}$ & $\mathrm{x}$ & $\mathrm{x}$ & & & & & & & & & 1,7 \\
\hline Peltophorum dubium (Spreng.) Taub. & & & $\mathrm{x}$ & $\mathrm{x}$ & $\mathrm{x}$ & & & & & & & & 2,4 \\
\hline Mimosaceae & & & & & & & & & & & & & \\
\hline Anadenanthera falcata (Benth.) Speg. & $\mathrm{x}$ & $\mathrm{x}$ & & $\mathrm{x}$ & $\mathrm{x}$ & & & $\mathrm{x}$ & & & & & 1,7 \\
\hline Inga sp. & $\mathrm{x}$ & $\mathrm{x}$ & & & & & & & & & & & 1,4 \\
\hline Mimosa sp. & & $\mathrm{x}$ & & & & & & & & & & & 0,1 \\
\hline Parapiptadenia sp. & & & $\mathrm{x}$ & $\mathrm{x}$ & & & & & & & & & 1,7 \\
\hline Leucaena leucocephala (Lam.) de Wit & & & $\mathrm{x}$ & & $\mathrm{x}$ & & & & & & & & 0,5 \\
\hline Pithecellobium sp. & & & & & $\mathrm{x}$ & & & & & & & & 0,1 \\
\hline Meliaceae & & & & & & & & & & & & & \\
\hline Cedrela sp. & & & $\mathrm{x}$ & & & & & & & & & & 1 \\
\hline Melastomataceae & & & & & & & & & & & & & \\
\hline Tibouchina sp. & $\mathrm{x}$ & & & & & & & & & & & $\mathrm{x}$ & 1,4 \\
\hline Myrtaceae & & & & & & & & & & & & & \\
\hline Eucalyptus sp.1 & $\mathrm{x}$ & $\mathrm{x}$ & $\mathrm{x}$ & $\mathrm{x}$ & $\mathrm{x}$ & $\mathrm{x}$ & $\mathrm{x}$ & $\mathrm{x}$ & $\mathrm{x}$ & $\mathrm{x}$ & $\mathrm{x}$ & $\mathrm{x}$ & 31,6 \\
\hline Eucalyptus sp.2 & & & & & $\mathrm{x}$ & $\mathrm{x}$ & $\mathrm{x}$ & $\mathrm{x}$ & $\mathrm{x}$ & $\mathrm{x}$ & & & 6,7 \\
\hline Eucalyptus sp.3 & & & $\mathrm{x}$ & & & & $\mathrm{x}$ & $\mathrm{x}$ & $\mathrm{x}$ & $\mathrm{x}$ & & & 8,2 \\
\hline Eugenia uniflora L. & & & $\mathrm{x}$ & & & & & & & & & & 0,1 \\
\hline Psidium guajava L. & & & $\mathrm{x}$ & & & & & & & & & & 0,4 \\
\hline Myrtaceae tipo 1 & $\mathrm{x}$ & & & & & & & & & & & & 0,4 \\
\hline Myrtaceae tipo 2 & $\mathrm{x}$ & $\mathrm{x}$ & $\mathrm{x}$ & $\mathrm{x}$ & $\mathrm{x}$ & & & & & & & & 1,4 \\
\hline Myrtaceae tipo 3 & & & & & $\mathrm{x}$ & $\mathrm{x}$ & $\mathrm{x}$ & $\mathrm{x}$ & & & & $\mathrm{x}$ & 5,2 \\
\hline Myrtaceae tipo 4 & $\mathrm{x}$ & & & & & & & & & & & & 0,1 \\
\hline Myrtaceae tipo 5 & $\mathrm{x}$ & & & $\mathrm{x}$ & $\mathrm{x}$ & $\mathrm{x}$ & $\mathrm{x}$ & & & $\mathrm{x}$ & $\mathrm{x}$ & $\mathrm{x}$ & 3 \\
\hline Myrtaceae tipo 6 & $\mathrm{x}$ & & & & & & & & & & & & 0,1 \\
\hline Myrtaceae tipo 7 & & & & & & $\mathrm{x}$ & $\mathrm{x}$ & & & & & & 0,9 \\
\hline Proteaceae & & & & & & & & & & & & & \\
\hline Macadamia sp. & & & & & & & $\mathrm{x}$ & & & & & $\mathrm{x}$ & 1 \\
\hline Rhamnaceae & & & & & & & & & & & & & \\
\hline Hovenia dulcis Thunb. & & $\mathrm{x}$ & $\mathrm{x}$ & & & & & $\mathrm{x}$ & & & & & 0,8 \\
\hline Rubiaceae & & & & & & & & & & & & & \\
\hline Rubiaceae tipo & & $\mathrm{x}$ & & & & & & & & & $\mathrm{x}$ & & 0,8 \\
\hline Coussarea curvigemmia Dwyer. & & & & & $\mathrm{x}$ & $\mathrm{x}$ & & & & & & & 0,4 \\
\hline Rutaceae & & & & & & & & & & & & & \\
\hline Citrus sp. & & & & & & & & & & & $\mathrm{x}$ & & 0,4 \\
\hline Salicaceae & & & & & & & & & & & & & \\
\hline Salix sp. & & & $\mathrm{x}$ & & & & & & & & & & 0,3 \\
\hline Sapindaceae & & & & & & & & & & & & & \\
\hline Serjania sp. & $\mathrm{x}$ & & & & & & & & & & & & 0,3 \\
\hline Solanaceae & & & & & & & & & & & & & \\
\hline Solanum sp. & & & & & & & & & & $\mathrm{x}$ & $\mathrm{x}$ & $\mathrm{x}$ & 4,1 \\
\hline Verbenaceae & & & & & & & & & & & & & \\
\hline Aloysia triphylla (L’Hér.) Britton & & & & $\mathrm{x}$ & & & & & & & & & 0,1 \\
\hline Grãos de pólen não identificados & & & & & & & & & & & & & \\
\hline Tipo 1 (subprolato, médio, tricolporado e rugoso) & & $\mathrm{x}$ & & & & & & $\mathrm{x}$ & & & & & 1,4 \\
\hline Tipo 2 (prolato, médio, triporado e rugoso) & & $\mathrm{x}$ & & & & & & & & & & & 0,4 \\
\hline $\begin{array}{l}\text { Tipo } 3 \text { (prolato-esferioidal, médio, trocolporado e } \\
\text { microequinado) }\end{array}$ & & & $\mathrm{x}$ & $\mathrm{x}$ & & & & & & & & & \\
\hline Tipo 4 (tétrade tetraédrica, média e psilada) & $\mathrm{x}$ & & & & & & & & & & $\mathrm{x}$ & & 0,4 \\
\hline Total \% & & & & & & & & & & & & & 100 \\
\hline
\end{tabular}




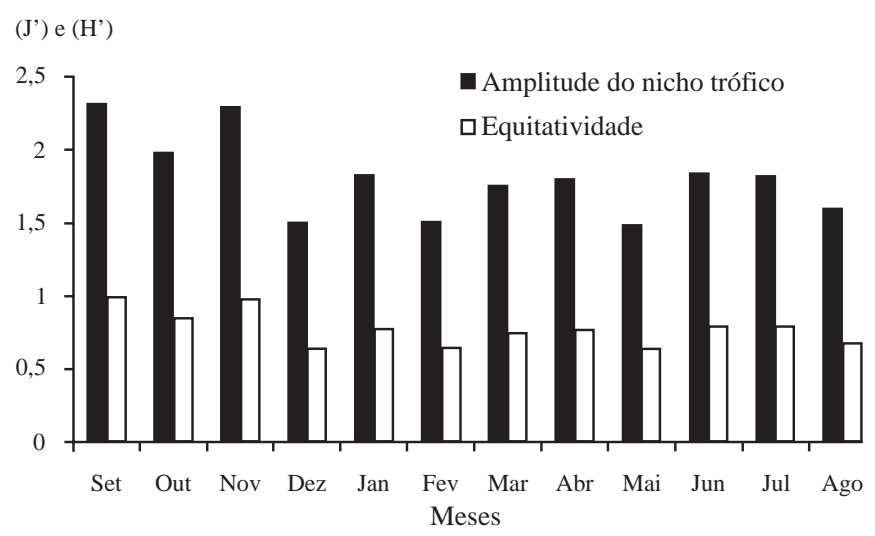

Fig. 1. Amplitude do nicho trófico (H') e equitatividade (J') para os tipos polínicos encontrados nas corbículas de Scaptotrigona depilis durante o período de Setembro de 2006 a Agosto de 2007 no Campus da UFGD, Dourados, MS.

a morfologia da abelha (tamanho do corpo, robustez da mandíbula e comprimento da probóscide) podem influenciar a escolha e exploração das fontes florais. Myrtaceae foi à fonte mais explorada e apresenta flores abertas com muitos estames, facilitando as visitas por $S$. depilis. A maioria das flores visitadas pelas abelhas possuía anteras de abertura longitudinal, onde o pólen ficava exposto e de fácil retirada. Uma exceção foi Solanum sp. que possui anteras tubulares e poricidas. Várias abelhas retiram o pólen desse tipo de antera por vibração buzz-pollination, porém nesse estudo não foram feitas observações em campo, e não se sabe se $S$. depilis é capaz de utilizar esse método de vibração das anteras, se faz perfurações para retirada do pólen ou se usa orifícios feitos por outras espécies.

Os valores de amplitude do nicho trófico de S. depilis atingiram índices mínimos em maio $\left(\mathrm{H}^{\prime}=1,49\right)$, dezembro $\left(H^{\prime}=1,50\right)$ e fevereiro $\left(H^{\prime}=1,51\right)$, meses com grande concentração de coletas de pólen em Myrtaceae. O valor máximo $\left(\mathrm{H}^{\prime}=2,32\right)$ foi obtido em setembro, seguidos pelos meses de novembro $\left(H^{\prime}=2,29\right)$ e outubro $\left(H^{\prime}=1,98\right)$ (Fig. 1), meses em que houve um considerável aumento

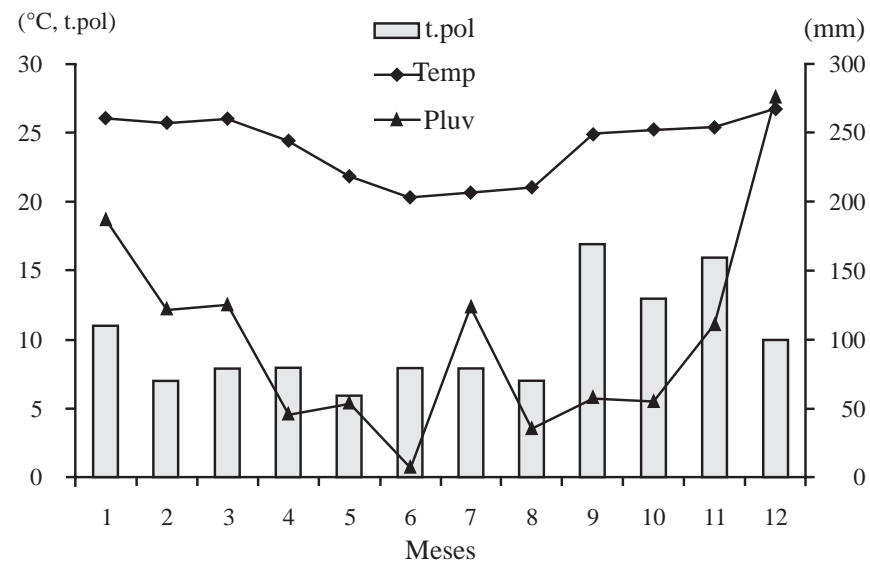

Fig. 2. Média mensal de temperatura $\left({ }^{\circ} \mathrm{C}\right)$, pluviosidade $(\mathrm{mm})$ e a relação com o número de tipos polínicos (t.pol) coletado por S. deplilis durante o período de setembro de 2006 a agosto de 2007. na temperatura e no volume pluviométrico (Fig. 2), e conseqüentemente uma maior variabilidade e disponibilidade de recursos, principalmente de espécies de Caesalpinaceae e Mimosaceae (Tabela I). Ramalho (1990) encontrou valores baixos de extensão do nicho trófico em Scaptotrigona, com predominância também de pólen de Eucalyptus spp. Estudando colônias de Melipona marginata marginata, Kleinert-Giovannini \& Imperatriz-Fonseca (1987) verificaram índices baixos com concentração em uma só planta, e visitas discretas a várias outras fontes de pólen.

Houve maior uniformidade (equitatividade) na exploração de fontes de pólen por $S$. depilis em setembro $\left(\mathrm{J}^{\prime}=1\right)$, quando também se observou a maior amplitude do nicho trófico (Fig. 1). Kleinert-Giovannini \& Imperatriz-Fonseca (1987) estudando colônias de Melipona marginata constatou que o mês de agosto foi o mais uniforme e teve também a maior amplitude do nicho trófico dessa espécie, concluindo assim, que quanto maior a amplitude do nicho maior a uniformidade nas coletas. Essa relação pode ser comprovada nos meses de maio e dezembro, quando $S$. depilis foi mais heterogênea em suas coletas $\left(\mathrm{J}^{\prime}=0,64\right)$, e também apresentou baixa amplitude de nicho trófico (Fig. 1).

De setembro a março, observou-se valores relativamente altos para temperatura (acima de $25^{\circ} \mathrm{C}$ ). Nesse período foram registrados os maiores picos de amplitude do nicho trófico. Já em dezembro foi verificado um decréscimo nas coletas dos grãos de pólen, quando o índice de pluviosidade chegou ao seu valor máximo (277 mm), limitando assim as saídas das campeiras para as coletas (Fig. 2). Esses fatores são determinantes para a atividade das abelhas quando chegam a valores extremos, dificultando sua atividade de forrageio.

A disponibilidade e localização dos recursos podem ter influenciado as escolhas de fontes de pólen de S. depilis, pois a abelha coletou principalmente pólen de Eucaliptus sp., que teve uma floração longa, com alta densidade floral, quando comparada com as outras espécies coletadas. Além disso, havia vários representantes de Myrtaceae a distâncias de 50 a 100 metros do ninho, que podem ter-se tornado atrativas pela economia de tempo de forrageio. De acordo com Gressler et al. (2006) Myrtaceae é uma das famílias apícolas mais importantes do Brasil, sendo citada em quase todos os estudos que envolvem a determinação das espécies vegetais de interesse para abelhas. Outras espécies vegetais conspícuas como $S$. glandulatum e $P$. dubium que floresceram por um período não tão longo como Eucalyptus, mas suas floradas abundantes foram, juntas, responsáveis por 5,6\% do total de polens coletados (Tabela I).

Foi verificada uma estreita relação de $S$. depilis com alguns grupos de vegetais, destacando-se em importância como fonte de pólen as famílias Myrtaceae (Eucalyptus spp.) e Mimosaceae para a manutenção da colônia ao longo do ano. Os altos valores de amplitude de nicho trófico e equitatividade ocorreram quando houve maior diversidade de espécies em floração, mostrando que $S$. depilis possui um potencial generalista em resposta à diversidade de fontes florais disponíveis. 
Agradecimentos. A Dra. Maria Lucia Absy pela identificação de alguns grãos de pólen e também pelo apoio na parte escrita, ao Dr. Odival Faccenda pelo apoio Estatístico e ao CNPq (Conselho Nacional de Desenvolvimento Científico e Tecnológico) pela concessão da bolsa de Pós-Graduação para realização do projeto (procs. 132302/2007-5).

\section{REFERENNCIAS}

Absy, M. L. 1975. Polén e esporos do Quaternário de Santos (Brasil). Hoehnea 5: $1-25$.

Absy, M. L.; J. M. F Camargo; W. E. Kerr \& I. P. A. Miranda. 1984. Espécies de plantas visitadas por meliponinae (Hymenoptera:Apoidea), para coleta de pólen na região do médio Amazonas. Revista Brasileira de Biologia 44: 227-237.

Empresa Brasileira de Pesquisa Agropecuária. Centro Nacional de Pesquisa de Solos.1999. Sistemas Brasileiro de Classificação de Solos. Brasília, $412 \mathrm{p}$.

Erdtman, G. 1952. Pollen Morphology and Plant Taxonomy. Angiosperms. Chronica Botanica Co., Waltham, Mass., 539 p.

Erdtman, G. 1960. The acetolysis method. A revided description. Upsala Svensk Botanisk Tidskrift 54: 561-564.

Gressler, E.; M. A. Pizo \& P. C. Morellato. 2006. Polinização e dispersão de sementes em Myrtaceae do Brasil. Revista Brasileira de Botânica 29: 509-530

Imperatriz-Fonseca, V. L.; A. Kleinert-Giovannini \& M. Ramalho. 1989. Pollen harvest by eusocial bees in a non natural community in Brazil. Journal of Tropical Ecology 5: 239- 242.

Imperatriz-Fonseca, V. L.; A. Kleinert-Giovannini; \& M. Ramalho. 1993. Flores e Abelhas em São Paulo. São Paulo, Edusp/FAPESP, 192 p.

Kerr, W. E.; V. A. Nascimento \& G. A. Carvalho. 1999. Preservation of native Brazilian bees: A question of historical and ecological concience. Ciência e Cultura 51: 390-393.

Kleinert-Giovannini, A. \& V. L. Imperatriz-Fonseca. 1987. Aspects of the trophic niche of Melipona marginata marginata Lepeletier (Apidae, Meliponinae). Apidologie 18: 69-100.

Matheson, A.; S. L. Buchmann; C. O’Toole; P. Westrich \& I. H. Williams. 1996. The Conservation Of Bees. Londres, Academic Press for The Linnean Sociey of London and the International Bee Research Association, $254 \mathrm{p}$.

Marques-Souza, A. C.; I. P. A. Miranda.; C. O. Moura; A. Rebelo \& E. M. Barbosa. 2002. Características morfológicas e bioquímicas do pólen coletado por cinco espécies de Meliponineos da Amazônia Central. Acta Amazônica 32: 217-229.

Marques-Souza, A. C.; M. L. Absy \& W. E. Kerr. 2007. Pollen harvest features of the Central Amazonian bee Scaptotrigona fulvicutis Moure 1964 (Apidae: Meliponinae), in Brazil. Acta Botânica Brasílica 21: $11-20$.

Nogueira-Neto, P. 1970. A Criação de Abelhas Indígenas sem Ferrão. 2a ed. Chácaras e Quintais. São Paulo. 365 p.

Pielou, E. C. 1977. Mathematical Ecology. 2a ed, New York, WilleyInterscience publication, John Willey Sons, 385 p.

Ramalho, M. 1990. Foraging by stingless bees of the genus Scaptotrigona (Apidae, Meliponinae). Journal of Apicultural Research 29: 61-67.

Ramalho, M.; A. Kleinert-Giovannini \& V. L. Imperatriz-Fonseca. 1989. Utilization of floral resources by species of Melipona (ApidaeMeliponinae). Floral preferences. Apidologie 20: 185-195.

Ramalho, M.; A. Kleinert-Giovannini \& V. L. Imperatriz-Fonseca. 1990. Important bee plants for stingless bees (Melipona e Trigonini) and africanized honey bees (Apis mellifera) in neotropical habitats: a Review. Apidologie 21: 469-488.

Ramalho, M.; L. S. Guibu.; T. C. Giannini; A. Kleinert-Giovannini \& V. L. Imperatriz-Fonceca. 1991. Characterization of some southern Brasilian honey and bee plants through pollen analysis. Journal of Apicultural Research 30: 81-86.

Ramalho, M.; M. D. Silva \& C. A. L. Carvalho. 2007. Dinâmica de uso de fontes de pólen por Melipona scutellaris Latreille (Hymenoptera: Apidae): Uma análise comparativa com Apis mellifera L. (Hymenoptera: Apidae), no domínio tropical Atlântico. Neotropical Entomology 36: 37-45.

Roubik, D. W. 1995. Pollination of Cultivated Plants in the Tropics. FAO Agric. Serv. Bull., 18. Rome: Food Argic. (Org.).196 p.

Roubik, D. W. \& J. E. Moreno. 1991. Pollen and Spores of Barro Colorado Island. Monographs in Sistematic Botany from the Missouri Botanical Garden. 36:1-300.

Salgado-Labouriau, M. L. 1973. Contribuição à palinologia dos cerrados. Rio de Janeiro. Academia Brasileira de Ciências, 285 p.

Shannon, C. E. \& W. Weaver. 1949. The Mathematical Theory Of Communication. Urbano, Univ. Illinois Press, 117 p.

Wilms, W. \& B. Wiechers. 1997. Floral resource partitioning between native Melipona bees and the introduced africanized honey bee in the Brazilian Atlantic rain forest. Apidologie 28: 339-355.

Zavatini, J. A. 1992. Dinâmica climática no Mato Grosso do Sul. Geografia. Rio Claro. IGCE/UNE 17: 65-91.

Recebido em 17/08/2008; aceito em 03/01/2010

Editor: Isabela Galarda Varassin 